\title{
Axonal injury in multiple sclerosis
}

\section{H Lassmann}

\section{Renewed interest in axonal destruction in MS}

M ultiple sclerosis (MS) is an inflammatory demyelinating disease, which implies that myelin sheaths are the primary target in the destructive process. However, this primary demyelinating process is inevitably associated with axonal injury and destruction, which is variable in its extent between different plaques of the same patients and even more variable between lesions of different patients. Axonal pathology has been noted in the earliest pathological descriptions of the disease and its cause and functional consequences have been discussed in detail in the early 20 th century. ${ }^{1}$ However, when it became clear from experimental studies that an inflammatory demyelinating disease similar to MS can be induced by autoimmunity against myelin antigens, the interest in axonal injury vanished. It only reappeared when recent MRI investigations provided increasing evidence for axonal and neuronal loss in MS brains. $^{1}$

Detailed quantitative studies on axonal injury and loss in MS appeared in the late 1990s. Using amyloid precursor protein (APP) as a marker for acute axonal injury Ferguson et al showed that massive axonal damage occurs during the stage of active demyelination in fresh lesions. $^{2}$ Similar conclusions were reached by Trapp et al studying axonal transsections in MS lesions by confocal laser microscopy. ${ }^{3}$ In addition, these studies confirmed earlier observations that acute axonal injury correlated with the degree of macrophage infiltration in the lesions and that macrophages were closely attached to damaged axons..$^{2-4}$ These basic observations were extended in several ways by Kornek et al. ${ }^{5}$ In this study the quantity of acute axonal injury was correlated with lesional stages, defined by the presence and antigenic composition of myelin debris in macrophages. These data suggested that massive acute axonal injury occurs during a small time window of about two weeks after onset of demyelination. In addition, there is a slow burning, ongoing axonal destruction, which can be seen even in inactive demyelinated plaques, in which inflammation is sparse or absent. Such ongoing axonal injury is lacking in remyelinated shadow plaques. ${ }^{5}$

These data, taken together, suggest that axons in MS lesions are destroyed in two different ways. During acute demyelination high numbers of axons are damaged, most likely by the action of toxic inflammatory mediators. This phase of massive axonal injury, however, lasts only for a few days to weeks. In contrast a low grade axonal degeneration occurs in silent inactive plaques. Although only few axons are destroyed at a given time point, such lesions may persist in the CNS for years. Thus, this low burning axonal injury may account more to the global axonal loss in MS than axonal degeneration in acute plaques and it may in part also explain the slow progression of clinical disability in the chronic progressive phase of the disease.

So far only limited data are available on the mechanisms of axonal injury and destruction. Not all axons are affected in the same way. Overall, small calliber axons are more vulnerable to damage in MS plaques compared with thick fibres. ${ }^{6}$ When considering the molecular mechanisms of axonal damage, two different phases of axonal disintegration have to be distinguished: the trigger of axonal damage and the downstream pathways of axonal dissolution (see fig 1). Although the triggers of axonal injury seem to be specific for inflammatory conditions, such as MS, the downstream mechanisms of axonal dissolution appear similar in a variety of different pathological conditions of the nervous system, including inflammation, ischemia, or trauma.

Axonal injury can be initiated through direct $\mathrm{T}$ cell mediated cytotoxicity. ${ }^{7}$ Class I MHC restricted $\mathrm{T}$ lymphocytes can transsect axons in vitro in an antigen dependent immunological reaction, ${ }^{8}$ and pathological studies revealed a weak correlation between tissue infiltration with cytotoxic $\mathrm{T}$ cells and acute axonal injury. ${ }^{9}$ The attachment of highly activated cytotoxic $T$ cells on demyelinated axons is frequently encountered in particular in lesions of Marburg's type of acute MS and the cytotoxic granules of such $\mathrm{T}$ cells are arranged in a polarised fashion towards the contact zone between the lymphocyte and the axon. Such a constellation suggests the specific interaction of the $\mathrm{T}$ cell with the target axon in the sense of an "immunological synapse". ${ }^{7}$ Even more important compared with direct $\mathrm{T}$ cell mediated cytotoxicity seems to be the interaction of activated macrophages or microglia cells with axons in the course of axonal injury. Such cells are consistently found in close contact with degenerating axons. Many of their toxic effector molecules may lead to axonal injury, although a direct injurious effect has so far only been shown for proteases ${ }^{10}$ and reactive nitrogen species. ${ }^{11}$ In particular, nitric oxide (NO) intermediates are particularly attractive candidates. At low concentration they may induce functional conduction block, thus explaining clinical deficit in the absence of overt structural damage. ${ }^{12}$ At higher concentrations, and in particular when axons are electrically active, NO derivatives may lead to irreversible destruction of axons. ${ }^{13}$ This may in part be accomplished by the blockade of mitochondrial function and the disturbance of energy metabolism, which can be induced by NO radicals. ${ }^{14}$ In addition, they may interfere with synaptic conduction and may even directly damage nerve cell bodies and dendrites. As nitric oxide intermediates can also be involved in demyelination and oligodendroglia damage, these molecules seem to be particularly attractive candidates in the pathogenesis of tissue damage in MS. ${ }^{11}$ In addition to toxins produced by $\mathrm{T}$ cells and macrophages, specific antibodies may be involved in the initiation of axonal injury. In other patients or lesions tissue damage occurs through a hypoxia like metabolic injury, which may be particularly effective in augmenting NO mediated axonal destruction. ${ }^{15}$

The trigger activates several downstream events, which in a cascade of action result in the final dissolution of the axon. A disturbance of axoplasmic membrane permeability and a state of relative energy failure leads to uncontrolled ion influx into the axoplasm, in particular in those that are still electrically active. ${ }^{13}$ Intra-axonal accumulation of sodium ions is then counteracted by a reverse operation of the sodium/calcium exchanger, resulting in excess intraaxonal $\mathrm{Ca}++$. This activates $\mathrm{Ca}++$ dependent proteases, which may degrade cytoskeletal proteins and, thus, impair axonal transport. Voltage gated calcium channels then accumulate at sites of disturbed axonal transport and become locally integrated into the axonal membrane. ${ }^{16}$ This may lead to further influx of $\mathrm{Ca}++$ into the axons, which finally will result in complete dissolution of the axonal cytoskeleton and axonal disintergration. This pathway can be inhibited for therapeutic purposes at several levels. Thus, NO mediated axonal injury can be inhibited by blockade of $\mathrm{Na}+$ channels or through an inhibitor of $\mathrm{Na}+/ \mathrm{Ca}++$ exchange $^{17}$ and $\mathrm{Na}+$ channel blockers have also been found neuroprotective in autoimmune encephalomyelitis in vivo. ${ }^{18}$ In addition, blockade of 


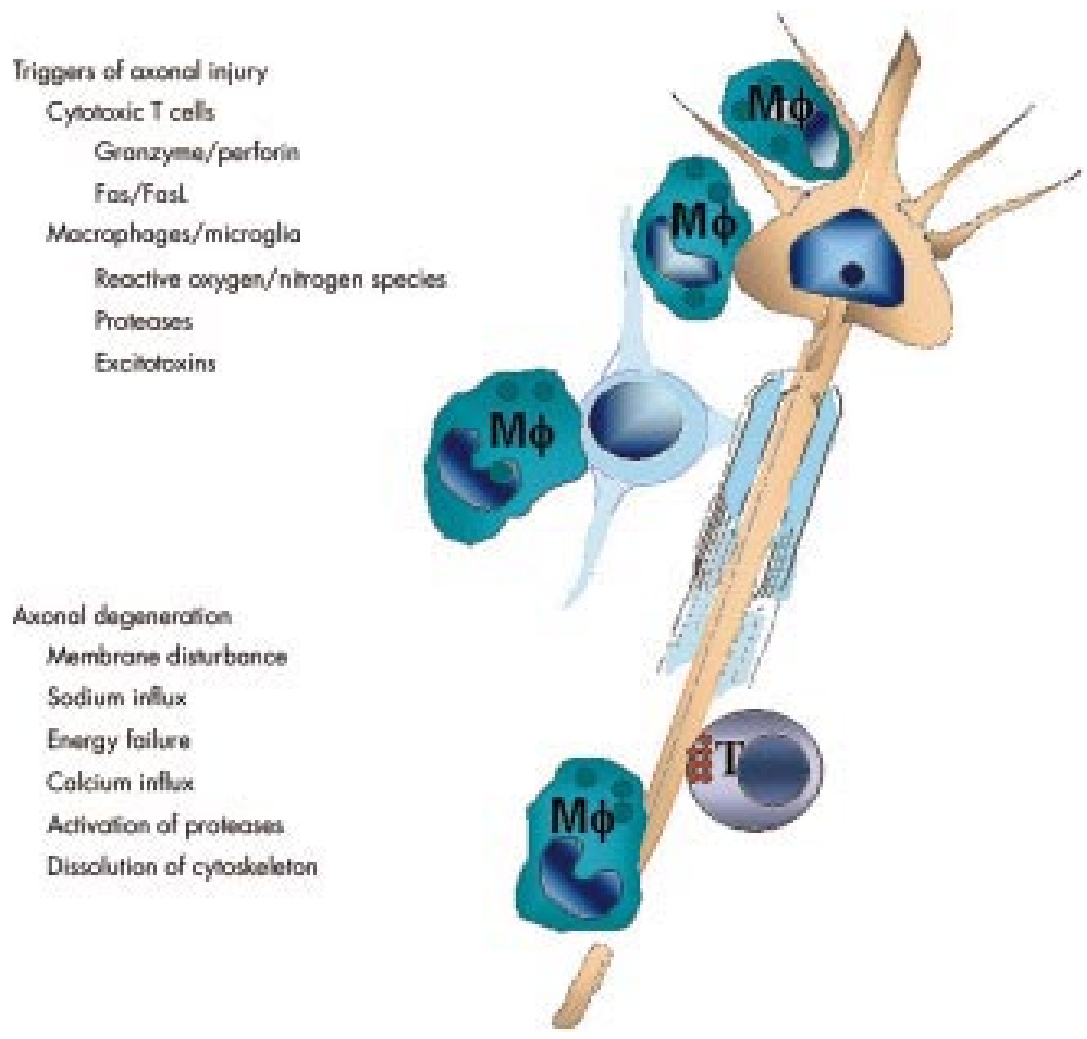

Figure 1 Triggers of axonal injury and axonal degeneration.

$\mathrm{N}$-type voltage gated $\mathrm{Ca}++$ channels too may ameliorate axonal injury. Finally, inhibition of $\mathrm{Ca}++$ dependent proteases can be used to prevent axonal injury. ${ }^{19}$ Interestingly, the same mechanisms of axonal disintergration seem to operate in ischemic conditions or in brain trauma. ${ }^{20-21}$ This raises hope that neuro and axon protective strategies, having been in development for brain ischemia for several years, may turn out to be equally effective in inflammatory brain diseases such as MS.

Although in relation to myelin sheaths axons are relatively spared in MS lesions, the degree of axonal destruction is profound. Its extent is highly variable, axonal density within plaques ranging $20-80 \%$ of that in the periplaque white matter. Within chronic established demyelinated plaques the reduction of axonal density is on average $60-70 \%$ compared with that in normal tissue of the same area. ${ }^{22} \mathrm{~A}$ similar percentage of axonal loss is found, when the absolute number of nerve fibres is counted in a defined tract area of the spinal cord. ${ }^{23}$ In contrast, in fresh lesions the reduction of axonal density is only in the range of $30 \%$. Two different factors seem to contribute to the increased axonal loss in chronic lesions: either the slow burning axonal disruption, which takes place chronically in established demyelinated plaques, or repeated demyelination and remyelination within the same area of the central nervous system.
Axonal destruction in demyelinated plaques results in widespread secondary "Wallerian" degeneration throughout the CNS tissue. This is most obvious when plaques are located in a defined tract system, where secondary degeneration is clearly visible distal to the plaque site. ${ }^{24}$ Secondary degeneration may lead in certain locations, such as the spinal cord to the paradox situation, that the loss of axonal profiles in a plaque is similar to that in the contralateral, seemingly "unaffected" white matter. 2325 Thus, in the spinal cord the net loss of axons in a tract system is the result of axonal injury in multiple demyelinated plaques located rostral or caudal from the site of investigation. Thus, secondary "Wallerian" degeneration is an important element, underlying diffuse abnormalities and axonal loss in the so called normal white matter, typically found in MS brains. ${ }^{26}$. In addition, however, there is a diffuse inflammatory process in the "normal" white matter of MS patients, which by itself is associated with bloodbrain barrier disturbance, brain oedema, ongoing axonal destruction, and reactive gliosis. These diffuse white matter changes seem to be particularly prominent in patients with primary progressive MS.

Axonal injury and loss in MS lesions has major consequences for the patients. Clinical deficit, induced by inflammation and demyelination, is principally reversible, while functional loss due to axonal degeneration overall is permanent. Yet the CNS has a large functional reserve capacity. Even in a tract system with clearly defined and measurable clinical function, such as the pyramidal tract, permanent clinical deficit is only seen when more than $60 \%$ of the axons in the tract system are lost. ${ }^{23}$ This implies that irreversible structural damage accumulates in MS brains already during the earliest stages of the disease, and permanent clinical deficit only appears when the functional reserve capacity of the CNS tissue is exhausted. Under these circumstances, axonoprotective therapy has to start early in the disease course in a preventive rather than a classical therapeutic strategy.

Its abundance, its early appearance in disease course, and the diffuse nature of axonal damage in MS, which is not necessarily restricted to demyelinating lesions, ${ }^{26}$ has triggered speculations that MS is a primary neurodegenerative disease, with a secondary (superimposed) inflammatory reaction, which may amplify the damage in the nervous system. Although such a scenario can so far not be definitely excluded, several observations argue against it. Inflammatory demyelination as a consequence of a neurodegenerative disease may eventually be discussed in adrenoleukodystrophy, but is not a feature of classical destructive processes of the CNS, such as neurodegenerative diseases, stroke, or trauma. In addition, in MS patients, dying early in the course of the disease or from fulminate Marburg's type of the disease, the pathological alterations are largely restricted to the demyelinated plaques and are always associated with inflammation. Similarly, the extent of axonal injury in old inactive demyelinated plaques, like that in acute lesions, correlates with the number of activated macrophages and microglia cells. ${ }^{5}$ Finally, diffuse white matter injury in MS is always associated with a profound and diffuse inflammatory reaction, mainly composed of cytotoxic $\mathrm{T}$ lymphocytes and activated microglia cells. Thus, the failure of current immunomodulatory or immunosuppressive therapies to effectively block disease in the progressive phase of MS may be more related to the poor efficacy of the anti-inflammatory therapies than to a non-inflammatory nature of the "neurodegenerative" component of MS.

\section{J Neurol Neurosurg Psychiatry 2003;74:695-697}

\section{Author's affiliation}

H Lassmann, Division of Neuroimmunology, Brain Research Institute, University of Vienna, Spitalgasse 4, A-1090 Wien, Austria;

hans.lassmann@univie.ac.at 


\section{REFERENCES}

1 Kornek B, Lassmann $\mathrm{H}$. Axonal pathology in multiple sclerosis: a historical note. Brain Pathol 1999;9:651-56.

2 Ferguson B, Matyszak MK, Esiri MM, et al Axonal damage in acute multiple sclerosis lesions. Brain 1997;1 20:393-9.

3 Trapp BD, Peterson J, Ransohoff RM, et al. Axonal transection in the lesions of multiple sclerosis. N Engl J Med 1998;338:278-85

4 Fraenkel M, Jakob A. Zur Pathologie der multiplen Sklerose mit besonderer Berücksichtigung der akuten Formen. Z Neurol 1913;14:565-603

5 Kornek B, Storch M, Weissert R, et al. Multiple sclerosis and chronic autoimmune encephalomyelitis: A comparative quantitative study of axonal injury in active, inactive and remyelinated lesions. Am J Pathol remyelinated lesions.

6 Evangelou N, Konz D, Esiri MM, et al. Size-selective neuronal changes in the anterior optic pathways suggest a differential susceptibility to injury in multiple sclerosis. Brain 2001; 124:1813-20.

7 Neumann H, Medana I, Bauer J, et al. Cytotoxic T lymphocytes in autoimmune and degenerative CNS diseases. Trend Neurosci 2002;25:313-9.

8 Medana I, Martinic MA, Wekerle $\mathrm{H}$, et al. Transection of MHC class l-induced neurites by cytotoxic T lymphocytes. Am J Pathol 2001;159:809-15.

9 Bitsch A, Schuchardt J, Bunkowski S, et al. Acute axonal injury in multiple sclerosis. Correlation with demyelination and inflammation. Brain 2000;1 23:1174-83.
10 Anthony DC, Miller KM, Fearn S, et al. Matrix metalloproteinase expression in an experimentally-induced DTH model of multiple sclerosis in the rat CNS. J Neuroimmunol 1998;87:62-72.

11 Smith KJ, Lassmann H. The role of nitric oxide in multiple sclerosis. Lancet Neurology $2002 ; 1: 232-41$

12 Redford EJ, Kapoor R, Smith KJ. Nitric oxide donors reversibly block axonal conduction: demyelinated axons are especially susceptible. Brain 1997;120:2149-57.

13 Smith KJ, Kapoor R, Hall SM, et al. Electrically active axons degenerate when exposed to nitric oxide. Ann Neuro 2001;49:470-9.

14 Bolanos JP, Almeida A, Stewart V, et al. Nitric oxide-mediated mitochondrial damage in the brain: mechanisms and implications fo neurodegenerative diseases. J Neurochem 1997;68:2227-40.

15 Aboul-Enein F, Rauschka H, Kornek B, et al. Preferential loss of myelin-associated glycoprotein reflects hypoxia-like white matter damage in stroke and inflammatory brain diseases. J Neuropathol Exp Neuro 2002;62:25-33.

16 Kornek B, Storch MK, Baver J, et al. Distribution of calcium channel subunit in dystrophic axons in multiple sclerosis and experiemntal autoimmune encephalomyelitis. Brain 2001;124:1114-24.

17 Kapoor R, Davies M, Blaker PA, et al. Blockers of sodium and calcium entry protect axons from nitric oxide-mediated degeneration. Ann Neurol 2003;53:174-80

18 Lo AC, Black JA, Waxman SG

Neuroprotection of axons with phenytoin in experimental allergic encephalomyelitis. Neuroreport 2002;28:1909-12.

19 Stys PK, Jiang Q. Calpain-dependent neurofilament breakdown in anoxic and ischemic rat central axons. Neurosci lett 2002;328: 150-4

20 Hewitt KE, Stys PK, Lesiuk HJ. The use-dependent sodium channel blocke mexiletine is neuroprotective against global ischemic injury. Brain Res 2001;898:281-7.

21 Wolf JA, Stys PK, Lusardi T, et al. Traumatic axonal injury induces calcium influx modulated by tetrodotoxin-sensitive sodium channels. J Neurosci 2001;15:1923-30.

22 Mews I, Bergmann M, Bunkowski S, et al. Oligodendrocyte and axon pathology in clinically silent multiple sclerosis lesions. Multiple Sclerosis 1998:4:55-62.

23 Biartmar C, Kidd G, Mork S, et al. Neurological disability correlates with spinal cord axonal loss and reduced $\mathrm{N}$-acetyl aspartate in chronic multiple sclerosis patients. Ann Neurol 2000:48:893-901.

24 Evangelou N, Konz D, Esiri MM, et al Regional axonal loss in the corpus callosum correlates with cerebral white matter lesion volume and distribution in multiple sclerosis. Brain 2000; 123: 1845-9.

25 Lovas G, Szilagyi N, Majtenyi K, et al. Axonal changes in chronic demyelinated cervical spinal cord plaques. Brain 2000;123:308-17.

26 Evangelou N, Esiri MM, Smith S, et al. Quantitative pathological evidence for axonal loss in normal appearing white matter in multiple sclerosis. Ann Neurol 2000;47:391-5.
It is clear that the clinical diagnosis of DLB is relatively common in this population, but it may be even more prevalent given that the consensus criteria used by McKeith et al have a relative low sensitivity for diagnosing this disease. ${ }^{4}$ The definition of dementia in this study was also based on consensus criteria together with the Mini-Mental State Examination scores, but with no systematic exclusion of other medical problems, using imaging or blood tests. Neuropathological confirmation of disease would obviously be useful, however the distinction between DLB and dementia in Parkinson's disease remains contentious, ${ }^{5}$ and so even if this study included postmortem analysis of the brains, issues of disease classification would remain. Indeed, the increased sensitivity to detect Lewy bodies using alpha-synuclein immunohistochemistry (which post dates the original consensus criteria of McKeith et $a l^{4}$ ) creates uncertainty even at postmortem.

Prevalence studies must balance the conflict that arises between (a) assessing large populations, thus enabling identification of larger numbers of affected patients, and (b) performing detailed evaluations or investigations of all identified individuals using qualified experts or trained personnel. With large population denominators, detailed assessments become impossible, and either screening tools or random sampling must be used, as has been done in the selection of the 700 individuals studied in this paper. 
There is always some concern that patients who refuse participation in descriptive studies may have differing rates of disease from those who do participate, which may result in less precise prevalence estimates being made. Unfortunately it seems that other sources of clinical data regarding patients refusing participation are absent in this study, thus preventing any form of sensitivity analysis.

Nevertheless, this study does highlight the advantage of targeting specific populations of people at highest risk of disease from which to estimate disease frequency. Using community based samples with a defined population denominator allows accurate identification of both incidence and prevalence figures. Clinic based series have inevitable biases in patient ascertainment, with ill defined population denominators and no means of quantifying missing cases. It is now important that other investigators delineate age specific community based figures for this condition in their populations.

J Neurol Neurosurg Psychiatry 2003;74:697-698

\section{Authors' affiliations}

R A Barker, T Foltynie, Cambridge Centre for Brain Repair and Department of Neurology, Forvie Site, Robinson Way, Cambridge CB2 2PY, UK

\section{Correspondence to: R A Barker} rab46@cus.cam.ac.uk

\section{REFERENCES}

1 Rahkonen T, Eloniemi-Sulkava U, Rissanen S, et al. Dementia with Lewy bodies according to the consensus criteria in a general population aged 75 years or older. J Neurol Neurosurg Psychiatry 2003;74:720-4.

2 Stevens $T$, Livingston $G$, Kitchen $G$, et al. Islington study of dementia subtypes in the community. Br J Psychiatry 2002;1 80:270-6. community. Br J Psychiatry 2002; 180:27
Yamada T, Hattori H, Miura A, et al. Prevalence of Alzheimer's disease, vascula dementia and dementia with Lewy bodies in a Japanese population. Psychiatry Clin Neurosci 2001;55:21-5

4 McKeith IG, Ballard CG, Perry RH, et al. Prospective validation of consensus criteria for the diagnosis of dementia with Lewy bodies. Neurology 2000;54:1050-8.

5 Sulkava R. Differential diagnosis between early Parkinson's disease and dementia with Lewy bodies. Adv Neurol 2003;91:411-3.

\section{NEUROLOGICAL STAMP}

\section{Félix Guyon 1831-1920}

G uyon was a native of the Island of Réunion. He emigrated to France, studied medicine in Paris, and became a surgeon at Hôpital Necker and professor of genitourinary surgery in Paris. Guyon was an outstanding urologist and a pioneer in prostatectomy and cystoscopy.

In 1861 Guyon presented a paper on the hand to the Anatomical Society in Paris. This was published in the bulletin of the society, entitled "A note of an anatomical arrangement specific to the anterior aspect of the wrist not previously described"1 Given the date of the publication and the initial of the author, one can only conclude that this was Félix Guyon of urological fame. In his paper Guyon describes "une petite loge intraaponévrotique". The groove or tunnel formed between the pisiform and the hamate and ligaments is now eponymously known as Guyon's canal and the findings associated with ulnar nerve entrapment at this site Guyon's syndrome. In 1979 Guyon was honoured on a stamp issued by France on the occasion of the 18th Congress of International Society of Urologists, in Paris. He is portrayed with catheters (Stanley Gibbons no 2323, Scott no 1652.) Réunion also issued his stamp, but it was never sold.

\section{Reference}

1 Guyon F. Note sur une disposition

anatomique propre g la face anterieure de la region du poignet et non encore, decrite. Bulletins da la Societe Anatomique de Paris, 2nd Series 1861;6:184-6.

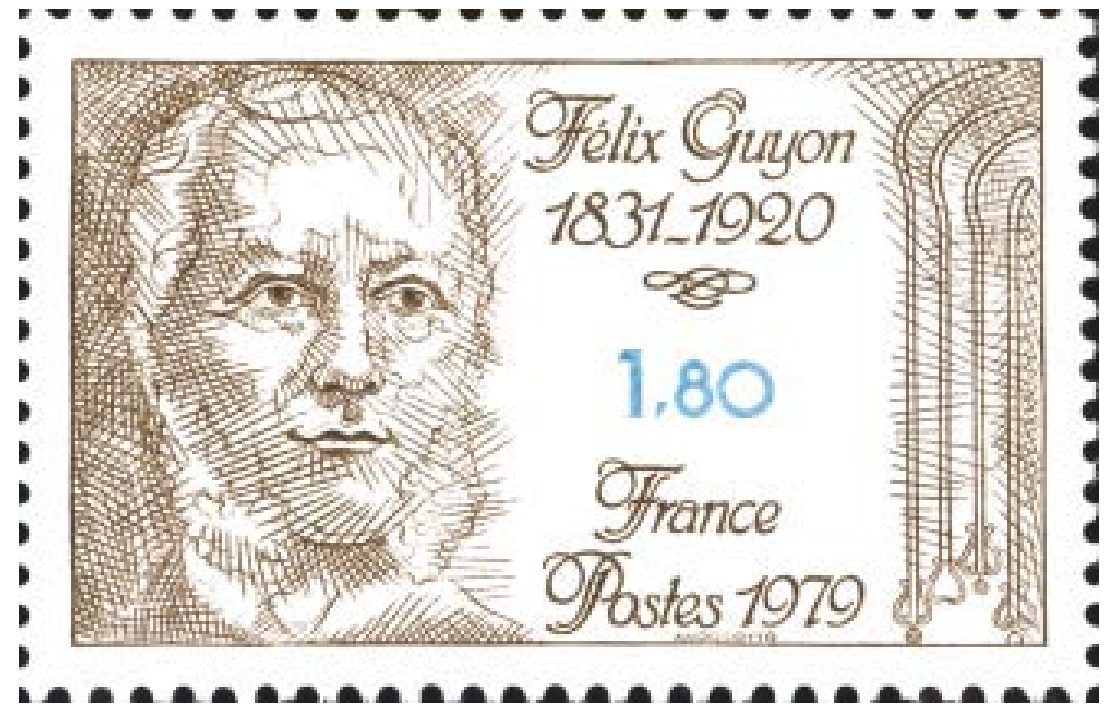

